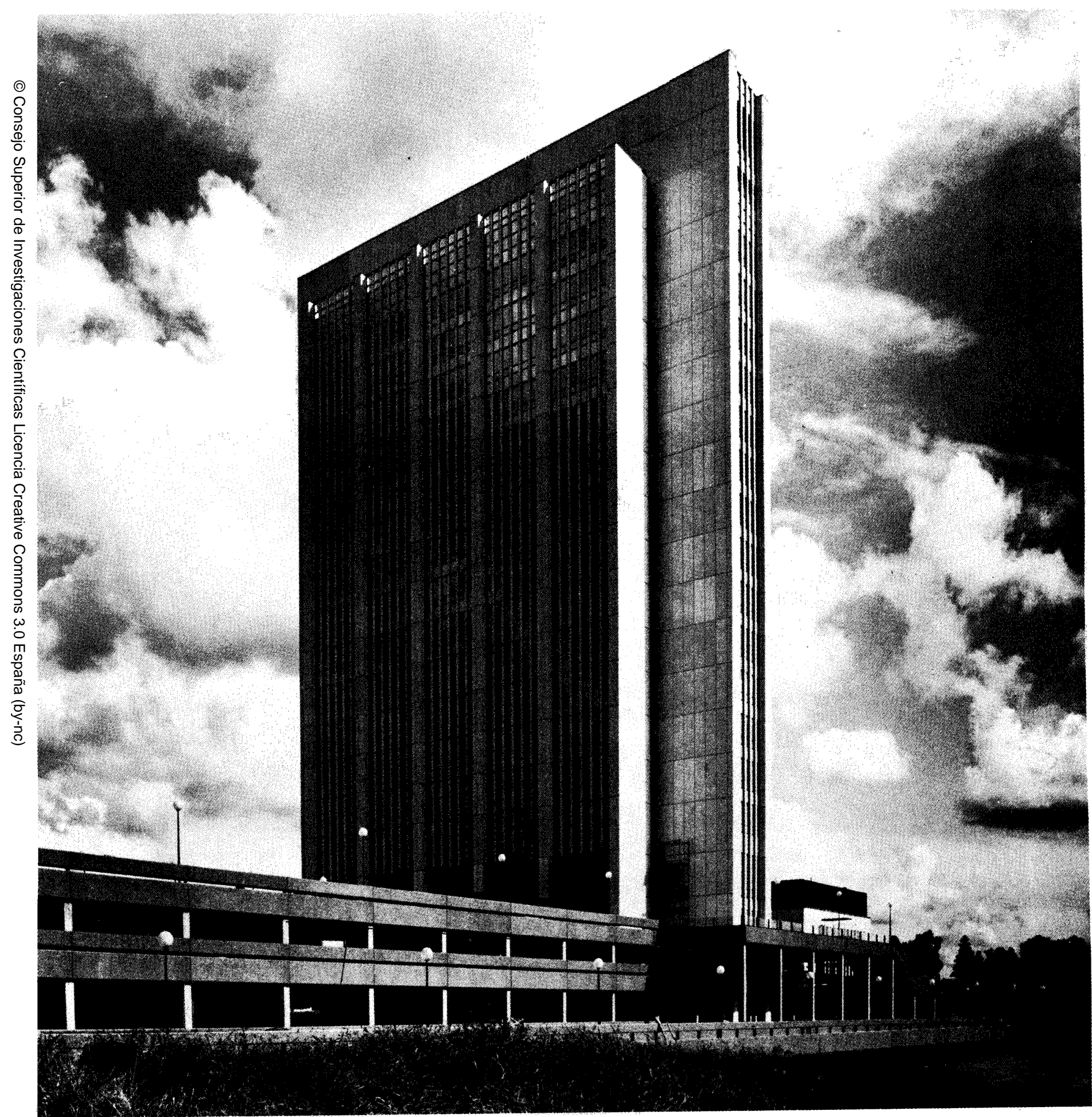

\title{
sinopsis
}

Este complejo, con un volumen total de $190.000 \mathrm{~m}^{3}$, está formado por tres edificios de características diferentes:

\section{sede central de la Compañía Neste Oy}

\section{Finlandia}

Castrén-Jauhiainen-Nuuttila, arquitectos

$131-143$

- una torre de $90 \mathrm{~m}$ destinada a oficinas, con las dos plantas superiores para instalaciones técnicas:

- un edificio de dos plantas con diversas áreas sociales: viviendas para vigilantes y personal de servicio, restaurante, centro de enseñanza y clínica:

- un aparcamiento de tres plantas con plazas para 500 vehículos.

El sistema estructural elegido para la torre fue el de una estructura metálica con forjados de hormigón aligerado.

Esta obra ha sido pionera en Finlandia, tanto en el campo de la construcción metálica, como en el de edificios de gran altura. 


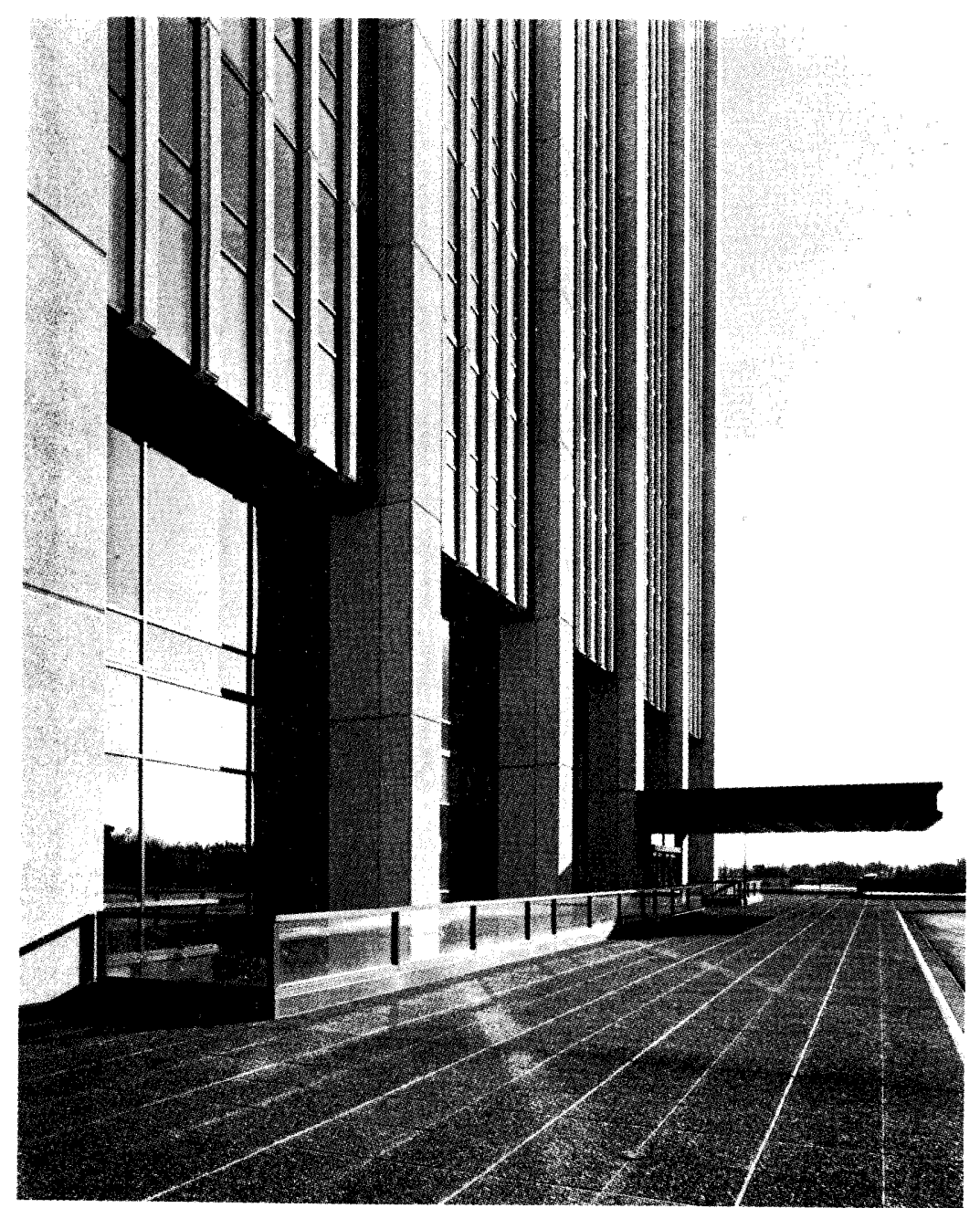

El complejo consta de tres bloques claramente diferenciados. El mayor, la torre de 90 metros de altura, con su eje en la dirección norte-sur, se equilibra y sujeta al suelo mediante otros dos edificios horizontales de dos plantas. El volumen total del complejo es de $190.000 \mathrm{~m}^{3}$.

Los tres bloques, en los cuales se ha conseguido que las distancias de circulación interna fuesen las mínimas posibles, tienen la distribución siguiente:

- La torre de 16 plantas se destina a oficinas, con capacidad total para 800 personas más un centro informático. Las dos plantas superiores sirven para las instalaciones técnicas.

(2)

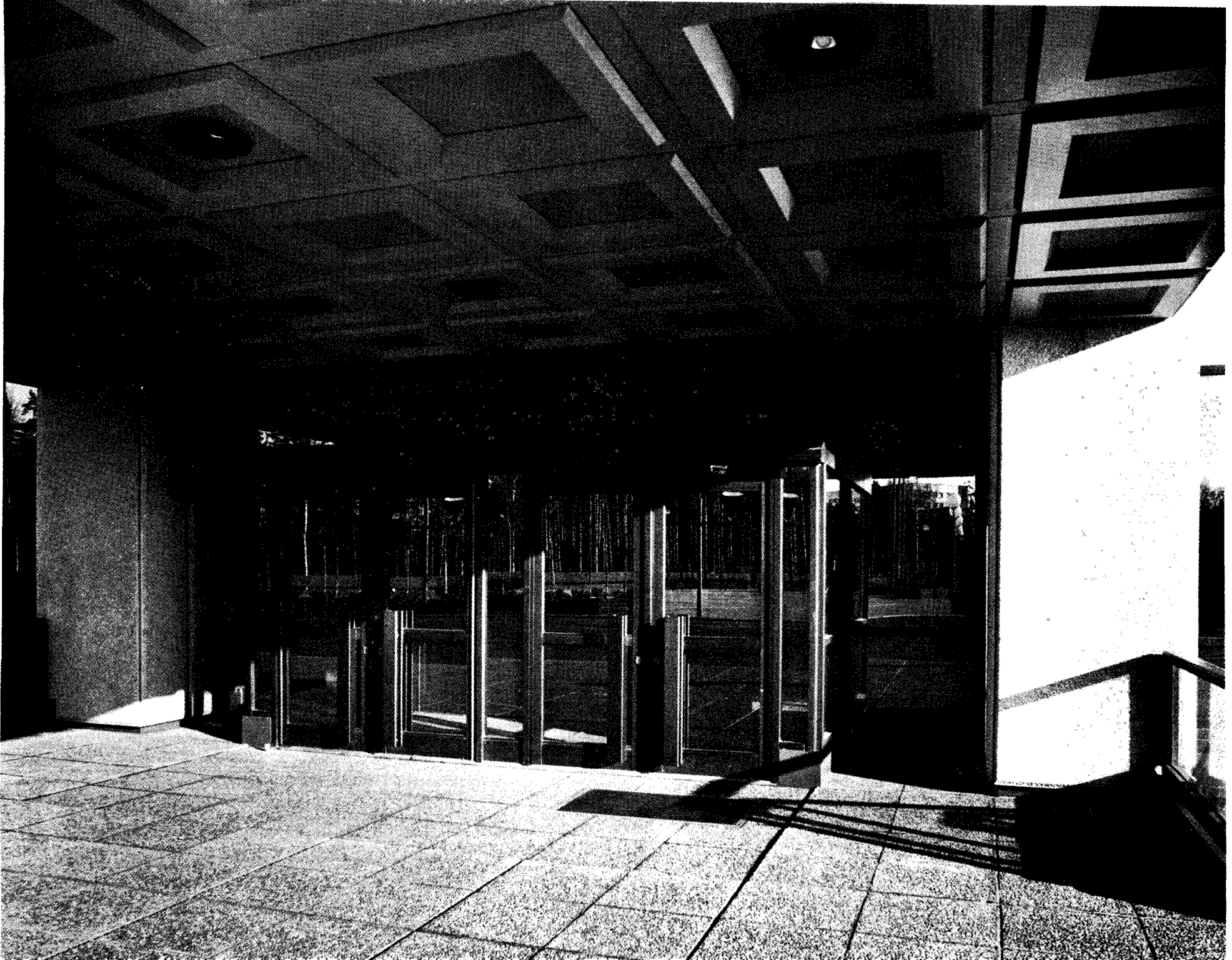




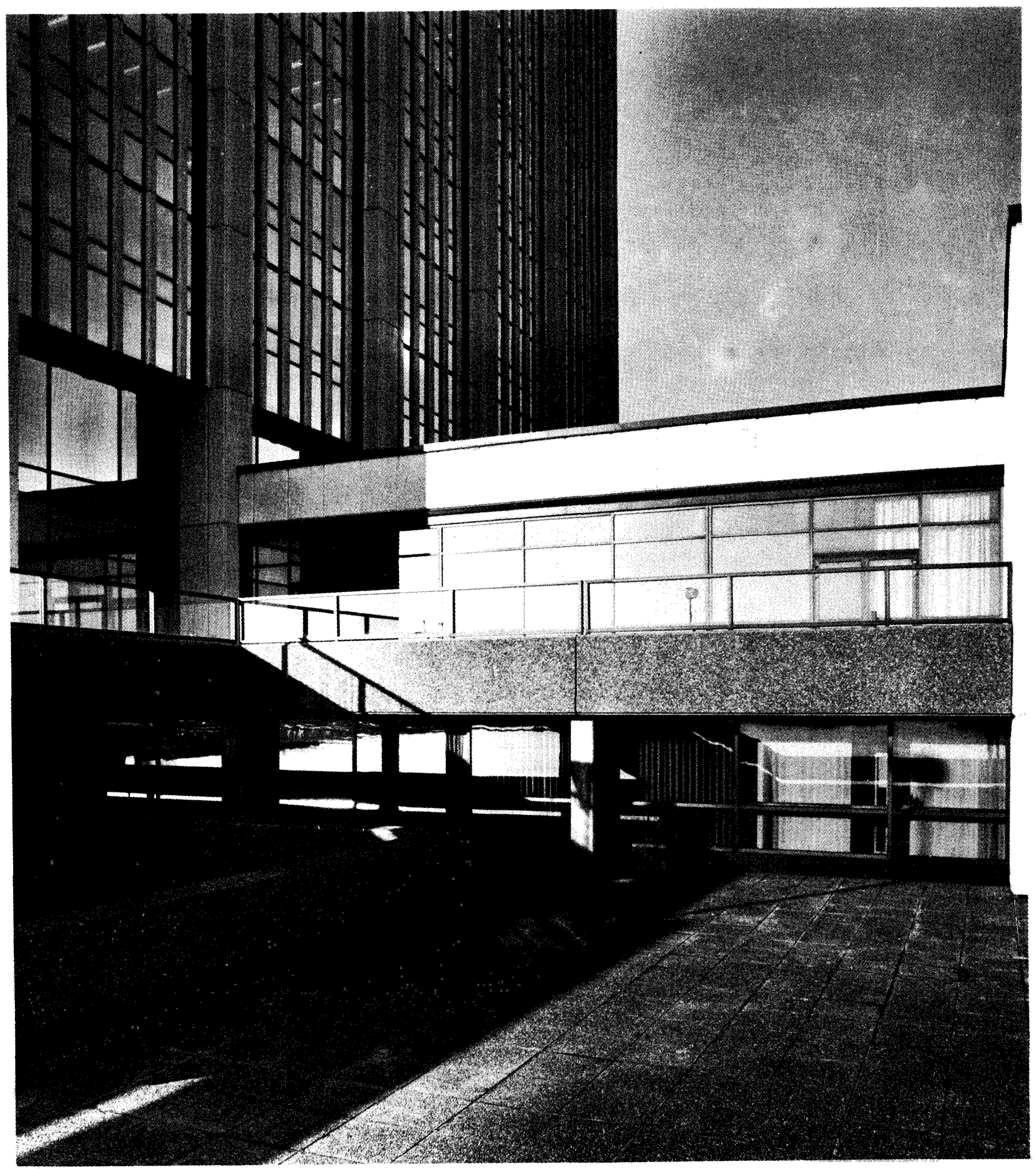

- El edificio situado al este de la torre consta, en su parte más cercana al mar, de viviendas para vigilantes y para personal de servicio así como lugares de recreo, mientras que en la parte contigua a la torre tiene un restaurante, un centro de enseñanza y una clínica. La existencia de estas áreas sociales son un factor muy importante en orden a conseguir un cierto bienestar para el personal. 


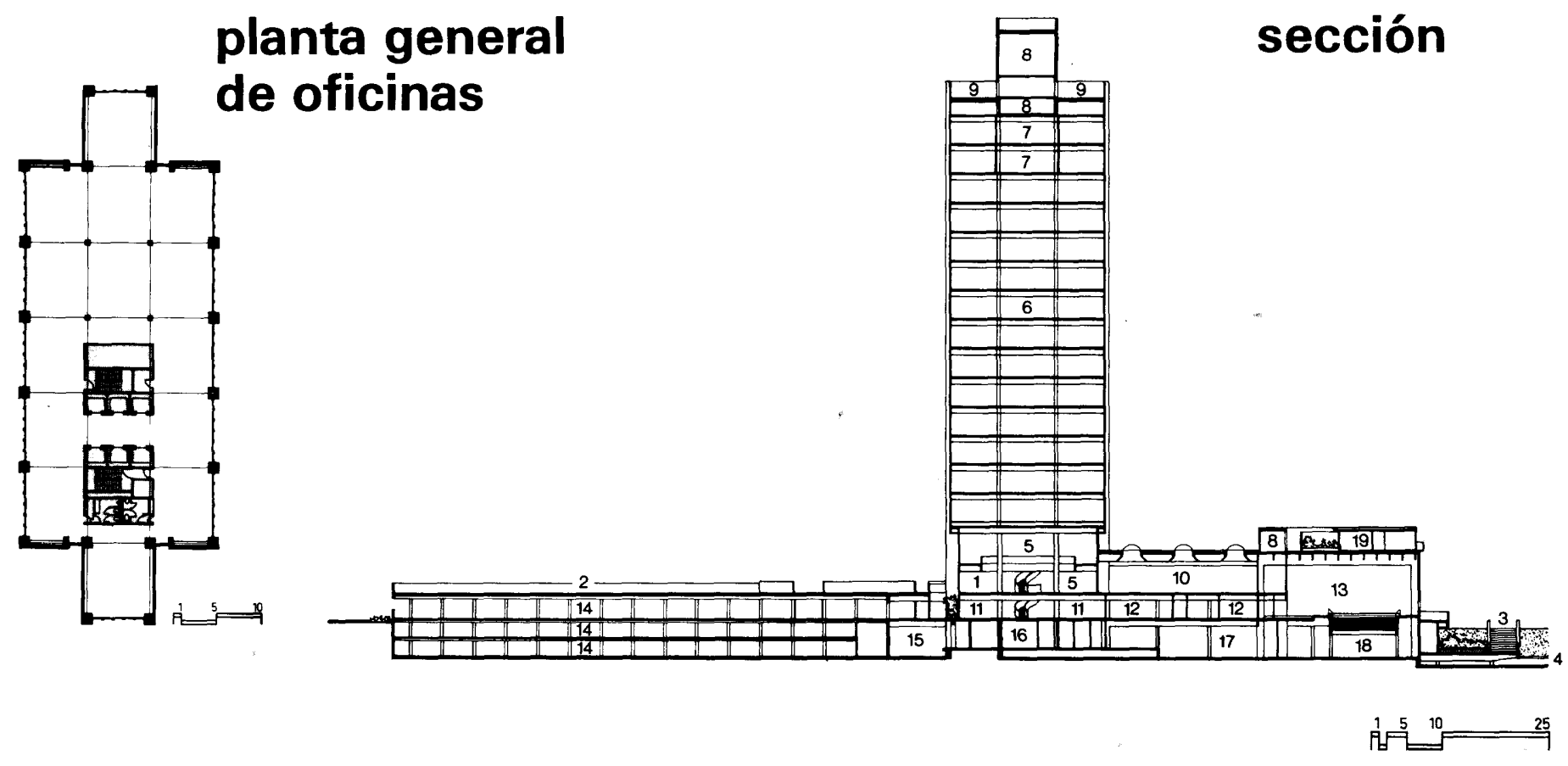

1. Entrada principal.-2. Nivel de la plaza.-3. Paseo de peatones.-4. Mar.-5. Hall principal.-6. Plantas de oficina.-7. Dirección.-8. Espacios técnicos (ventila ción, ascensor y sala de máquinasl.- -9. Terrazas.-10. Co medor.-11. Vestuario de personal. -12. Salas de aseo y vestuario sauna.-13. Piscina.-14. Aparcamiento.15. Zona de sauna. - 13. Piscina.-14. Aparcamiento.nes.-17. Instalaciones técnicas.-18. Archivos.-19. nes.-17. Instalaciones técnicas.-18. Archivos.-19.
Paso para el personal de servicio--20. Auditorio.-21. Salas de lectura y estudio.-22. Gimnasio.-23. Cocina.

\section{planta general}

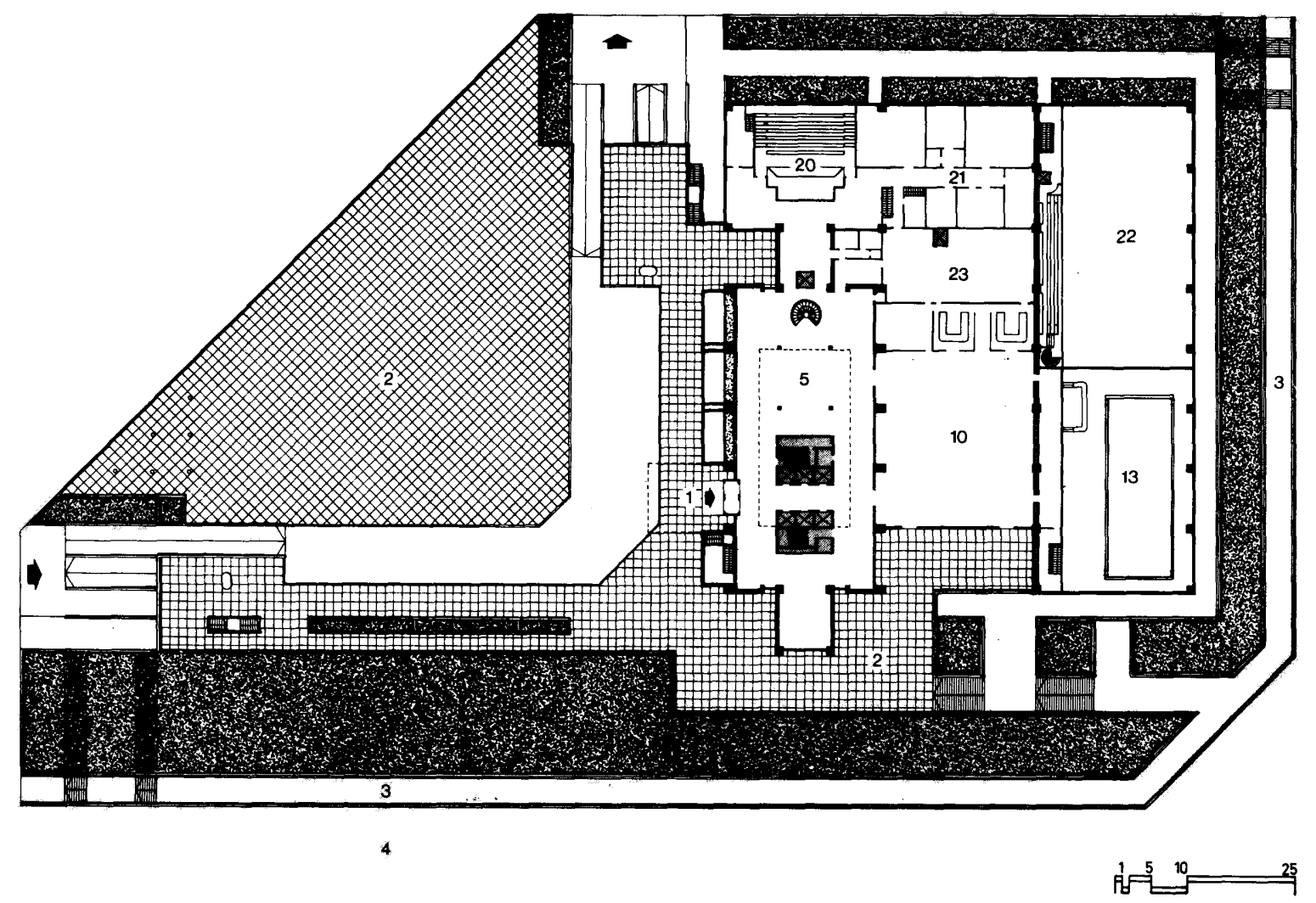




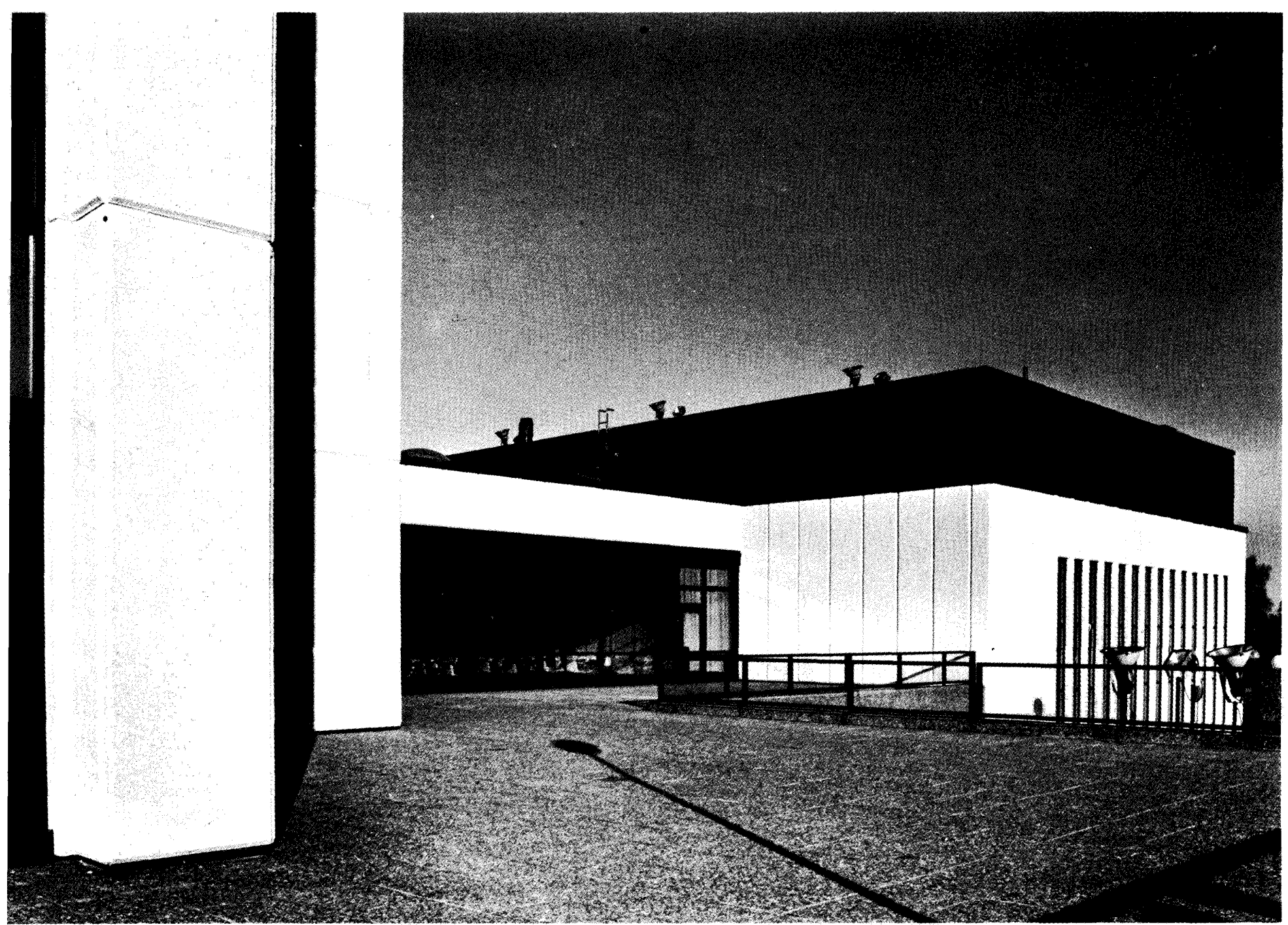

- El bloque al este de la torre tiene tres plantas de estacionamiento con plazas para 500 vehículos. La forma y disposición del aparcamiento permite la aproximación de los coches al complejo.

El vestíbulo de entrada tiene una altura de dos plantas con objeto de conseguir una circulacion fluida y agradable en el núcleo del complejo. Dicho vestibulo está rodeado por una galería para exposiciones de arte. Enlazados directamente con él están el auditorio, las salas de conferencias, el centro de enseñanza y el restaurante para el personal.

La altura de las plantas de la torre es de $4 \mathrm{~m}$, con lo que se obtiene una altura de $3 \mathrm{~m}$ en las habitaciones. Esta altura se consideró la más apropiada visualmente para las grandes oficinas de espacio abierto, que proporcionan un confortable entorno de trabajo. El espacio en las plantas se puede también adaptar a una distribución en corredor con compartimentos de trabajo aislados. 


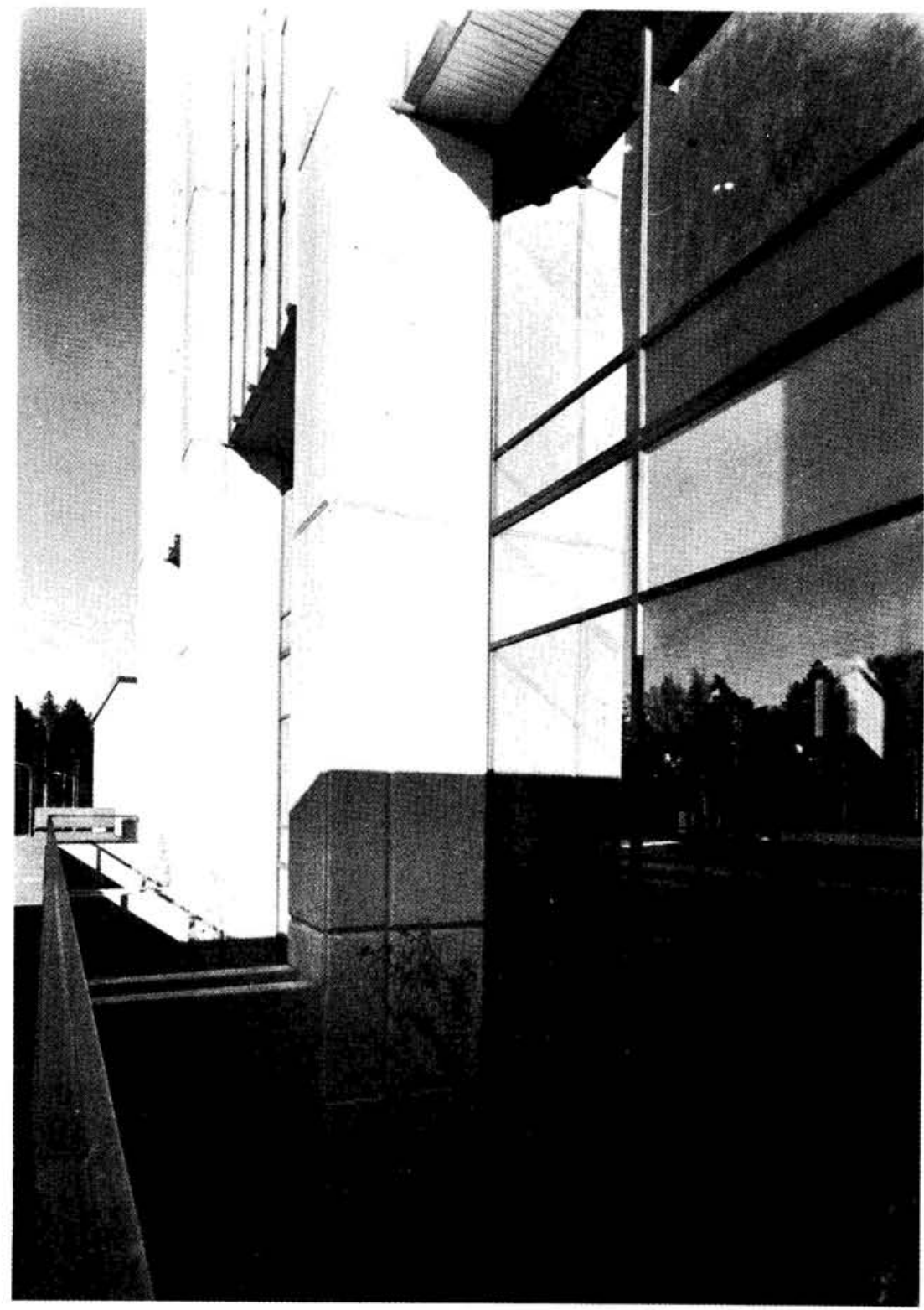

La torre está formada por una parte central rectangular flanqueada por dos planos rectangulares. Las fachadas de la parte central se realizaron con elementos de hormigón, mientras que los planos que la flanquean llevan cristales reflectores del calor, de color azulado, divididos por elementos más reducidos de hormigón. Todos los elementos de hormigón tienen un acabado de textura blanca. El efecto que produce este conjunto azul-blanco es el de lograr una conjunción total con el mar. En el revestimiento de la terraza inferior y de las pasarelas peatonales, que tienen una función independiente de las otras zonas, se usó un agregado de color pardo

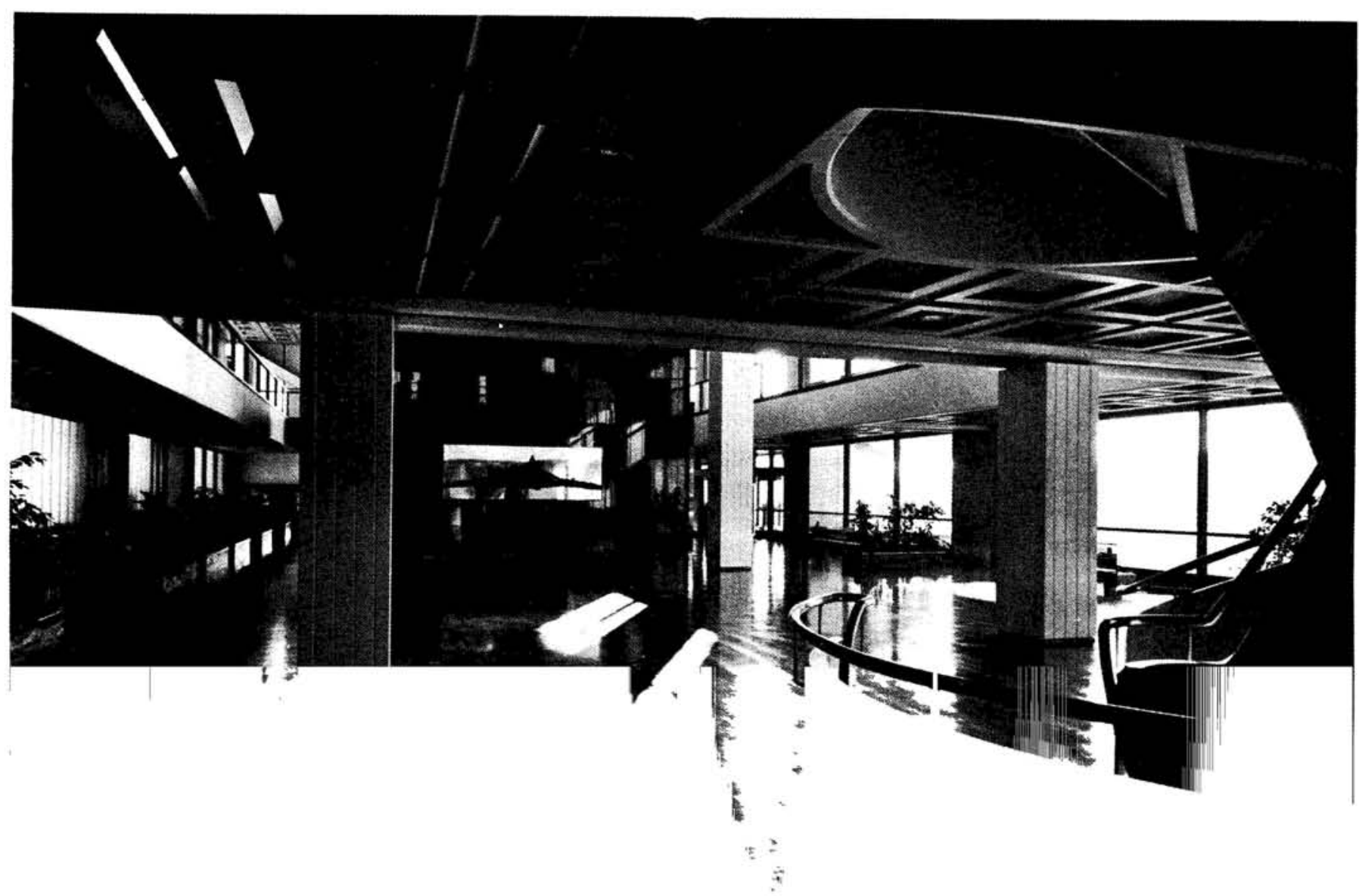


Desde el punto de vista arquitectónico, algunas de las razones por las que se eligió para el complejo de oficinas una torre de estructura metálica fueron:

- En un lugar costero, tan limitado como éste, una primera agrupación de muchos edificios de pequeño volumen destruirá parte del bosque y del carácter de la zona.

- Con una solución de edificio de gran altura y con parte del lugar construido sobre el mar se podrá a la vez ahorrar una gran parte de superficie de suelo natural y obtener paseos interesantes a la orilla del mar.

- Los promotores tenian una actitud favorable a que se construyese una solución metálica.

- Los proyectistas pretendieron obtener un espacio visualmente abierto a la vez que reducir volúmenes mediante una construcción ligera de acero.

\section{SISTEMA ESTRUCTURAL}

El esquema arquitectónico divide el complejo de la oficina central en tres bloques separados. El proyecto se realizó de forma que en el sentido estructural cada bloque funcionase como una entidad separada obteniéndose, de esta manera, un sistema económico y racional. Otras razones que también ayudaron a seleccionar el sistema estructural fueron: la disposición del conjunto de los edificios, su construcción sobre un suelo pobre y el hecho de que la mitad de la superficie esté sobre el mar y la necesidad de minimizar el trabajo.

\section{Cimentaciones}

La Oficina Central de Neste se cimentó sobre un emparrillado de hormigón armado. El emparrillado se apoya sobre pilotes de diferentes alturas $(3$ a $11 \mathrm{~m}$ ) que distribuyen las cargas directamente sobre la roca.

\section{Edificios más bajos}

Los pilares y vigas de estos edificios son de hormigón moldeado in situ. Las losas de hormigón pretensado aligerado se utilizaron para el forjado de las plantas intermedias. Para la cubierta de la piscina y de la sala de atletismo se usaron vigas postensadas. Para la cubierta de las ocho viviendas del personal de servicio se empleó hormigón ligero.

\section{Aparcamiento}

Los pilares del aparcamiento, las rampas y la base de la torre son de hormigón moldeado in situ. Los forjados intermedios constan de vigas-cajón de acero y losas de doble T apoyadas en el cordón inferior.

\section{Torre de oficinas}

Dos cajas de ascensores, de hormigón, construidas con encofrados deslizantes forman el núcleo de la torre de oficinas. Estas rigidizan la estructura de acero durante el montaje y actúan una vez acabado el edificio como su arriostramiento básico. Vigas trianguladas de acero y muros de cortante completan el efecto de arriostramiento.

Los pilares y las vigas-cajón principales se fabricaron de chapa de acero y se colocaron soldadas en secciones bastante largas. Los pilares y las cajas de ascensores llevan unas cartelas de acero donde se colocan simplemente apoyadas las vigas-cajón. El cordón inferior más ancho soporta las losas de hormigón ligero utilizadas para el forjado de las plantas intermedias.

Debido a esta disposición las vígas de acero y el resto de los componentes de los forjados tienen la misma altura estructural. Esto permite un gran margen de libertad en el proyecto de las redes eléctricas, de ventilación y de aire acondicionado, y simplifica su instalación.

Los pilares exteriores, en forma de cajón, miden $900 \times 900 \mathrm{~mm}^{2}$ y llevan conductos de aire acondicionado de $700 \mathrm{~mm}$ en su interior.

Los conductos adyacentes transportan aire frío o caliente. El aire mezclado, hasta la temperatura deseada, sale por la parte inferior del pavimento. Los pilares interiores de $400 \times 400$ milímetros cuadrados no tienen conductos.

Las conexiones de la estructura metálica se hicieron mediante juntas bulonadas. Sin embargo las conexiones de los pilares interiores van soldadas aunque se utilizaron pernos de montaje. 
Las columnas se realizaron para poder resistir durante 2 horas los ensayos al fuego, a la temperatura crítica de $300{ }^{\circ} \mathrm{C}$. Para la protección exterior de estos pilares se utiliza lana mineral mientras que el aislamiento interior se consiguió llenando el hueco existente entre el conducto interior y las paredes del pilar con hormigón ligero de materiales calizos.

Las columnas-cajón exteriores se pintaron con silicato de cinc para protegerlas contra la corrosión. El hormigón calizo protege el interior de la coIumna. Las estructuras de acero, situadas en el interior del edificio, no llevan este tipo de protección.

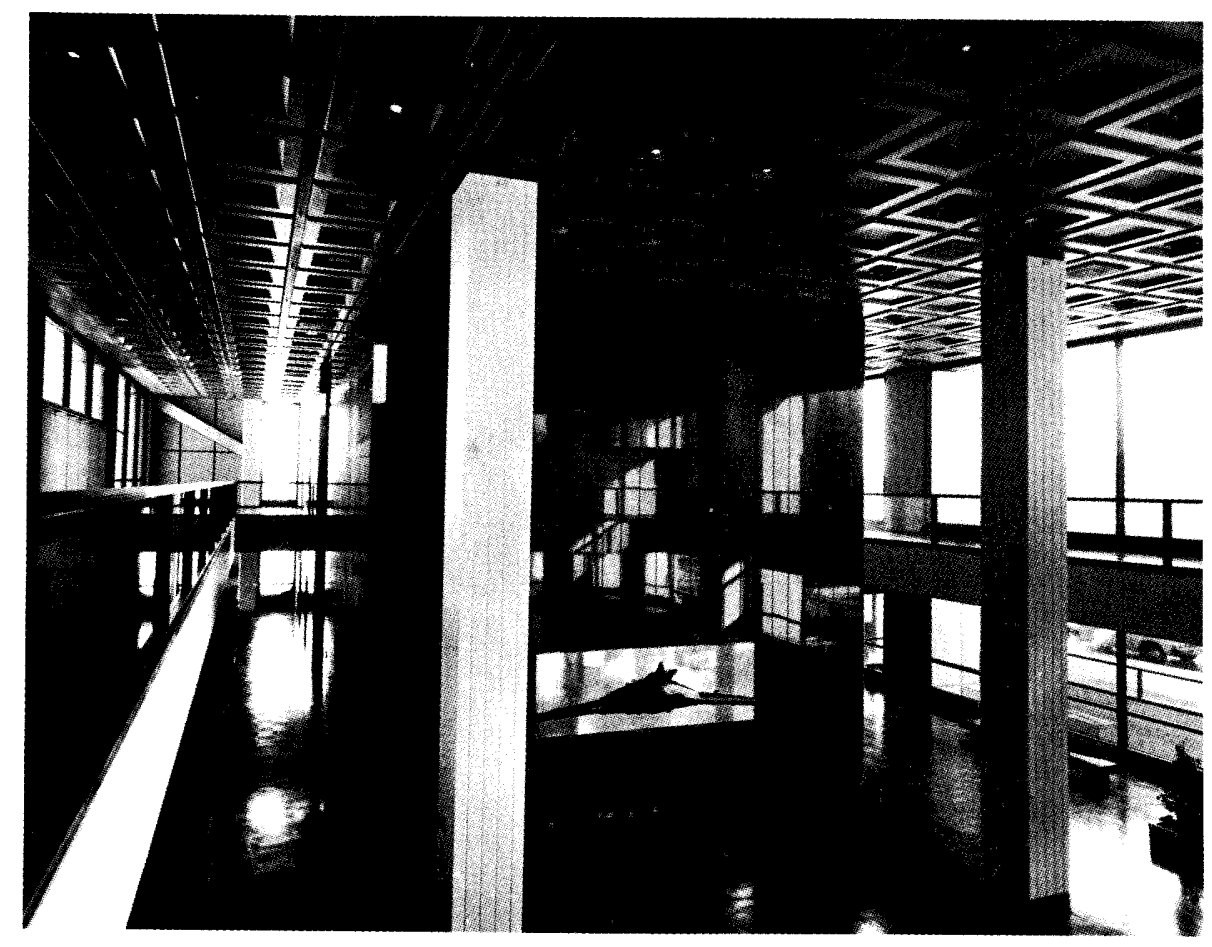

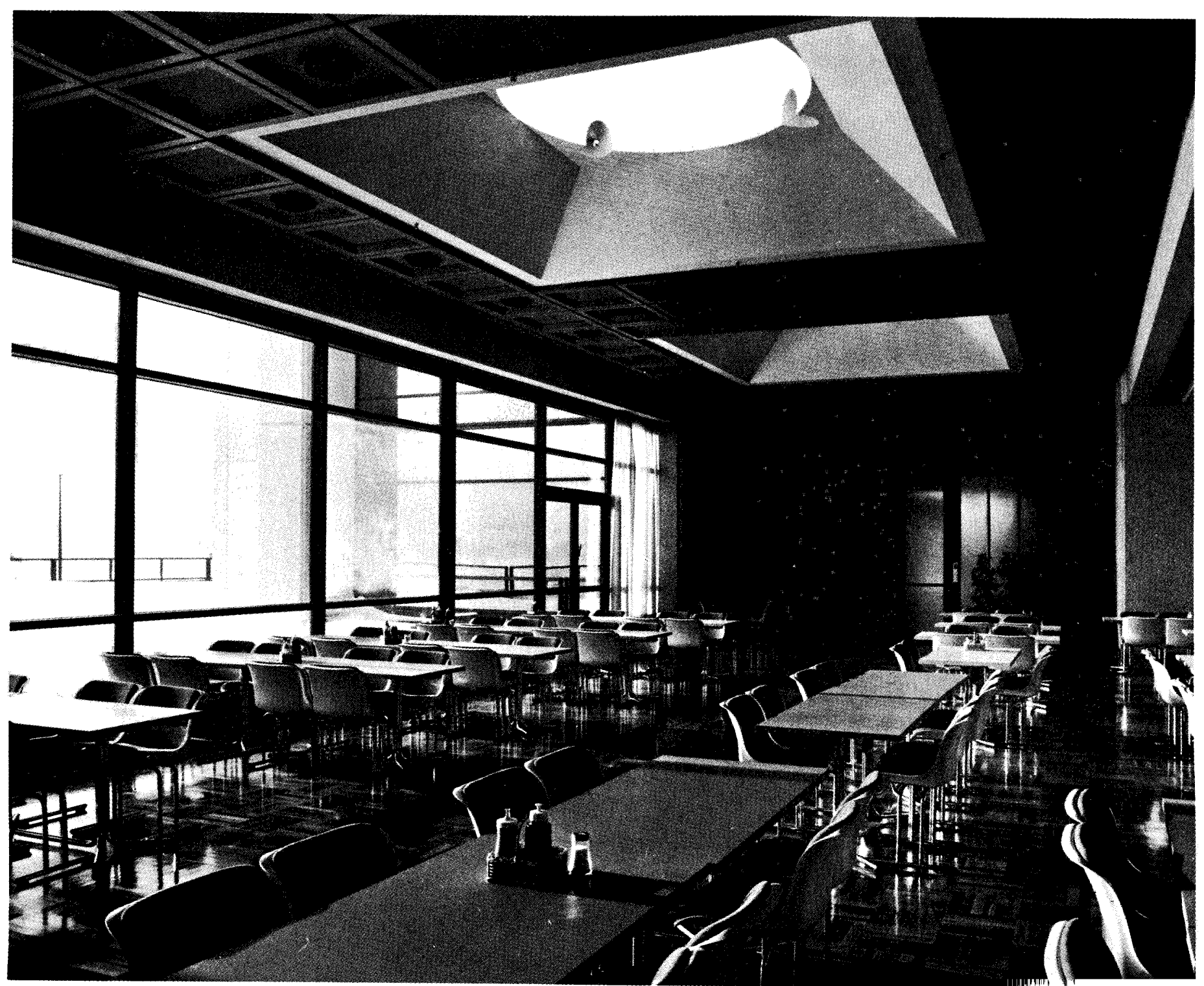




\section{Montaje de la estructura metálica}

El trabajo de montaje fue muy exigente. Como el edificio se encuentra parcialmente situado sobre el mar y la construcción se hizo durante los meses de invierno hubo que tener en cuenta los efectos considerables de viento y nieve. Por estas razones se hizo en el suelo toda la prefabricación que fue posible. Por ejemplo, las columnas se montaron en secciones con una longitud igual a la altura de tres plantas incluyendo los conductos de aire acondicionado con sus salidas.
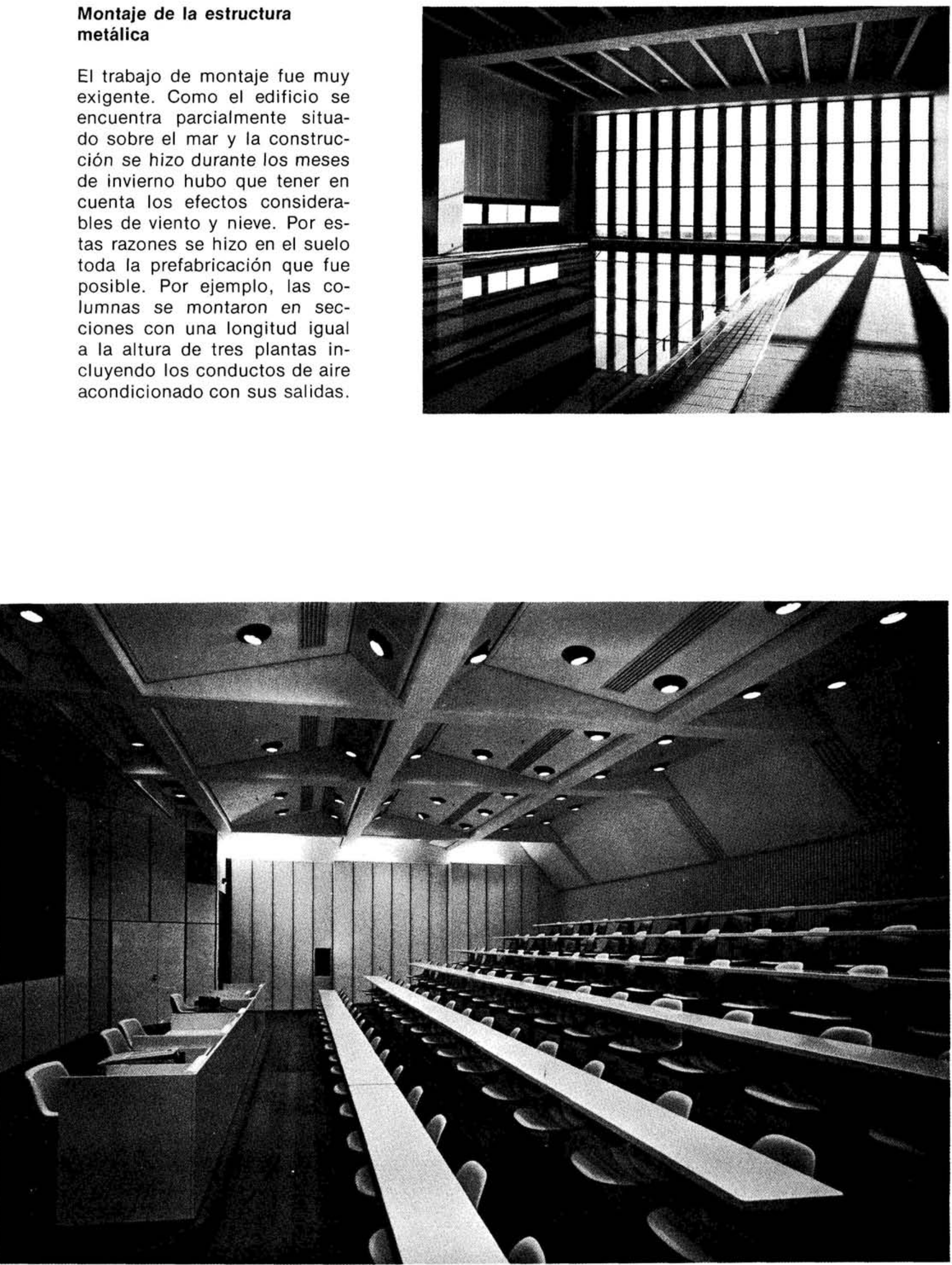
La elevación de los distintos elementos se hizo con la misma grúa-torre utilizada para los demás trabajos.

La instalación de las losas de hormigón aligerado para el forjado de cada planta se hizo en fase con el montaje de la estructura metálica.

El montaje de la estructura metálica y la realización de las demás construcciones se hizo también en fase. Para el montaje de cada suelo se necesitó un tiempo mínimo de 4 días

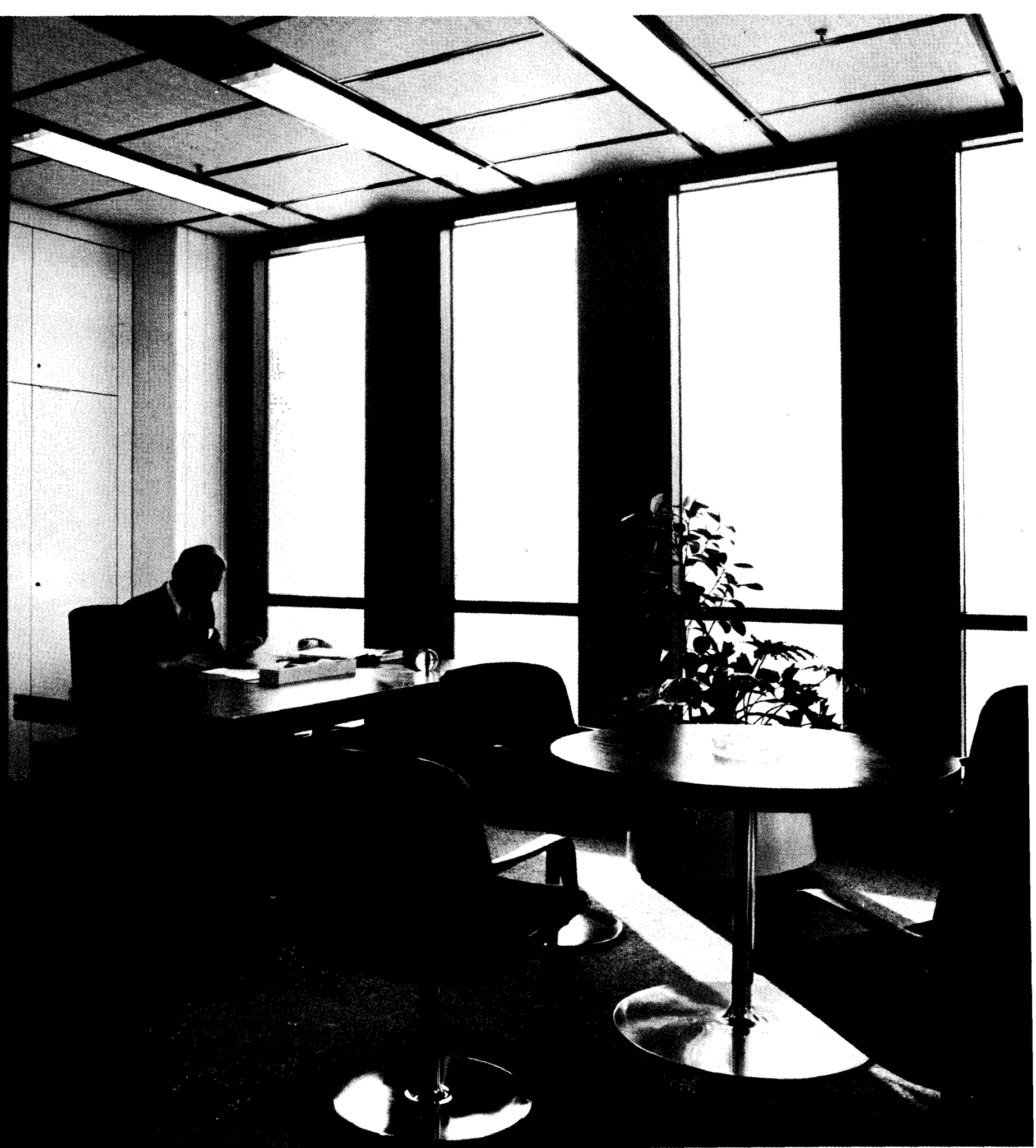



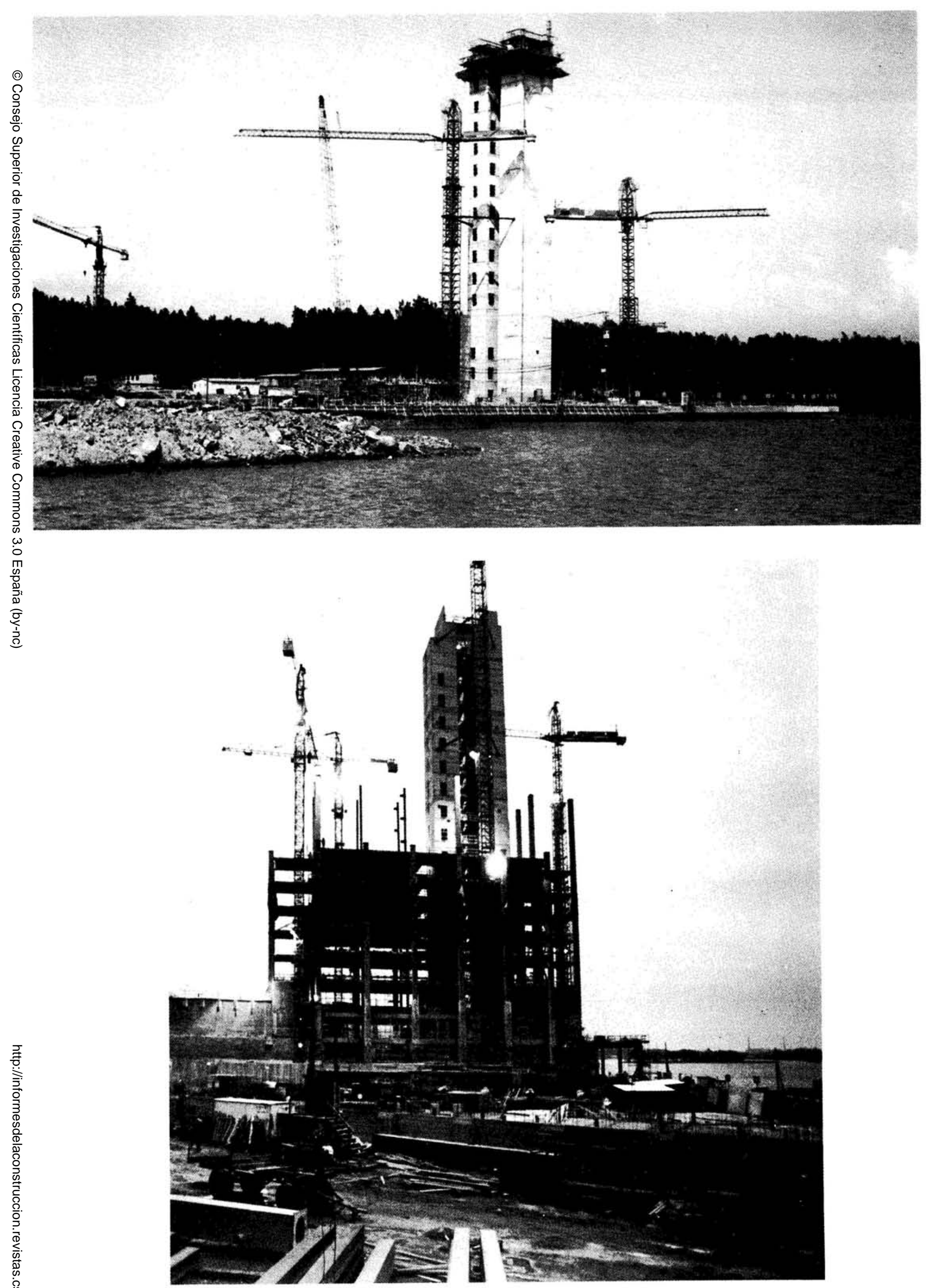


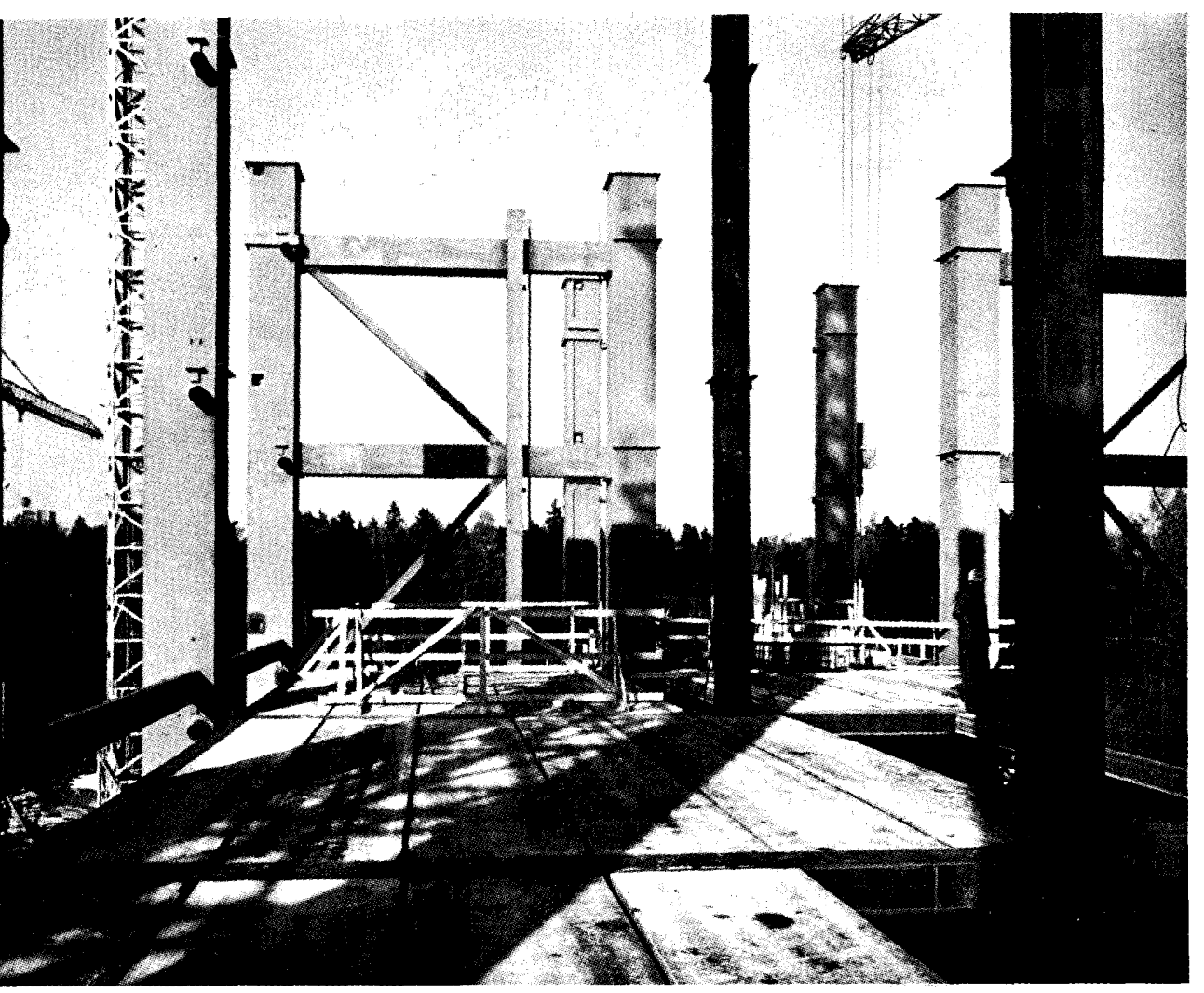

FOTOS: SEPPO PALMUNEN Y SIMO RISTA

\section{CONSECUENCIAS}

\section{Perspectivas futuras}

La oficina central de Neste es, en muchos aspectos, una de las obras de mayor extensión y de las más exigentes en el campo de la construcción metálica realizadas en Finlandia. Esta técnica, sólo en los últimos años, ha alcanzado un desarrollo importante en dicho pais. Este complejo es también pionero en la construcción de edificios de altura en Finlandia. Se

ha conseguido experiencia en este tipo de obra, con lo que se ha puesto la base para posteriores trabajos de este tipo. Ha servido igualmente para la puesta en marcha de normas reguladoras contra el fuego y de normas de seguridad para los usuarios de esta clase de edificios.

\section{Económicas}

Esta obra resultó económica por las siguientes razones:

- La construcción metálica permitió una mayor interacción en la planificación de las diversas disciplinas.

- La existencia de un programa de edificación que permitió el montaje de la estructura metálica sin interrupción durante los meses de invierno.

- El alto grado de exactitud conseguido en el dimensionamiento debido a ser un programa de construcción metálica.

- Beneficios adicionales tales como versatilidad, rapidez de construcción y espacio más aprovechado conseguidos por la misma razón: ser una construcción metálica.

\section{résumé}

SIEGE CENTRAL DE LA COMPAGNIE NESTE OY - FINLANDE CASTREN-

Castrén-Jauhiainen-Nuuttila, architectes

Cet ensemble, dont le volume est de $190.000 \mathrm{~m}^{3}$, est constitué par trois bâtiments de caractéristiques différentes:

une tour de $90 \mathrm{~m}$, destinée à des bureaux avec les deux niveaux supérieurs abritant les installations techniques

un bâtiment à deux étages avec diverses zones sociales: logements pour les surveillants et le personnel de service, restau rant, établissement d'enseignement et clinique;

un parking à trois niveaux pour 500 vé hicules.

Le système structural choisi pour la tour a été celui d'une structure métallique avec des planchers en béton léger.

Cet ouvrage a été le pionnier en Finlande, tant dans le domaine de la construction métallique que dans le domaine des bâtiments très élevés.

\section{summary}

HEAD OFFICES OF NESTE OY COM

PANY-FINLAND

Castrén-Jauhiainen-Nuuttila,

architects

This complex, with a volume of $190.000 \mathrm{~m}^{3}$ is made different characteristics

one $90 \mathrm{~m}$ tower used for offices, with the upper two floors for technical installations;

One two storey building with several social areas: living quanters for watchkeepers and service staff, restaurant, teaching centre and clinic;

a three storey car-park with capacity for 500 vehicles.

The structural system chosen for the tower was a metal structure with light weight concrete forgings.

This has been a pioneer work in Finland regarding both the metal construction and high buildings.

\section{zusammenfassung}

HAUPTSITZ DER GESELLSCHAFT NESTE OY-FINNLAND

Castrén-Jauhiainen-Nuuttila,

Architekten

Dieser Komplex, der ein Bauvolumen von $190.000 \mathrm{~m}^{3}$ aufweist, besteht aus drei Gebäuden verschiedener Merkmale:

ein $90 \mathrm{~m}$ hoher Geschäftsturm, dessen zwei oberste Stockwerke für technische Anlagen vorgesehen sind;

ein zweistöckiges Haus mit verschiedenen Sozialbereichen: Wohnungen für Wacht und Bedienungspersonal, Gaststätte, Shule und Klinik;

ein dreistöckiges Parkhaus mit Parkplätzen für 500 Fahrzeuge.

Das für den Turm gewählte Bausystem bes teht in einer Metallstruktur mit LeichtbetonFachwerken.

Dieses Bauwerk war sowohl hinsichtlich der Metallkonstruktion wie auch des Baues von Hochhäusern bahnbrechend in Finnland. 